\title{
The challenge in measuring low-permeability materials: a comparison of different approaches
}

\author{
Ben Laurich \\ Federal Institute for Geosciences and Natural Resources (BGR), Hannover, Germany \\ Correspondence: Ben Laurich (ben.laurich@bgr.de)
}

Published: 10 November 2021

\begin{abstract}
The German repository site selection procedure calls for a radioactive waste containment zone with a low-permeability host rock $\left(k_{f}<10^{-10} \mathrm{~m} \mathrm{~s}^{-1}\right.$, StandAG $\left.\$ 23,5\right)$ and long-term sealing by barrier materials (EndlSiAnfV, 2020; ESK, 2019). The potential host rocks, clay and rock salt, as well as the considered barrier materials, bentonite and compacted crushed salt, show permeability in the range of $k_{f} \sim 10^{-16} \mathrm{~m} \mathrm{~s}^{-1}$ ( $K \sim$ $10^{-21} \mathrm{~m}^{2}$ ). These low values suggest that advective flow is as slow as diffusive mass flux. Measuring such low permeability with adequate accuracy challenges measurement setups and respective error evaluation.

Methodologies. Several low-permeability measurements are carried out by transient tests, e.g. by monitoring controlled fluid pressure changes in: (1) pressure decay and (2) oscillating pulse tests. The first method (1) deviates permeability from the time needed to compensate pressure differences through the sample. The latter (2) monitors phase shift and amplitude attenuation of controlled pressure pulses passing through the sample. Any permeability measurement needs to be post-processed, e.g. for: (1) material-intrinsic controls (saturation state, storativity, the fluids' compressibility, etc.), (2) environmental controls (temperature, confining pressures, etc.) and (3) theoretical considerations (Klinkenberg correction, multi-phase wetting angles, etc.).

Salts. A porosity-permeability relation was found down to $K=10^{-19} \mathrm{~m}^{2}$ (e.g., Popp et al., 2007). Testing fluids were $\mathrm{NaCl}$ brine, oils, $\mathrm{He}$ and $\mathrm{N}_{2}$ as a fluid. As a matter of current research, a critical, low-permeability value might be associated with the so-called "percolation threshold" that defines the minimal requirements for an interconnected pore system (e.g., DAEF, 2016).

Clays. A major challenge is the long duration of sample saturation (up to several months) and pressure equilibration (often days), as well as precise, temperature-compensated measuring and the determination of the samples' storativity (e.g., Winhausen et al., 2021). Testing fluids are commonly designed mixtures mimicking the rocks' pore waters.

Geotechnical barrier materials. The permeability testing performed is similar to that of salt and clays mentioned above. However, both barrier materials, crushed salt and bentonite, have significant permeability early after emplacement. This is beneficial, as it allows the outflow of unwanted canister corrosion gases. Eventually, the permeability drops by orders of magnitude and the barriers become tight seals in the long-term. Here, identifying the gas entry/breakthrough pressure has been valuable (e.g., Rothfuchs et al., 2007).

Figure 1 shows a preliminary sensitivity analysis as an example of pressure decay measurements. It suggests that the pressure equilibration term (c), and hence the test duration, is most sensitive to the calculation of low permeability. However, the large variation of (representative) material and environmental controls make permeability measurements complex. This workshop aims to encourage discussions on uncertainty and sensitivity of the influencing controls, such that it may lead to a "best-practice" guide for permeability measurements.
\end{abstract}

Kurzfassung. Das deutsche Standortauswahlverfahren für ein Endlager fordert einen einschlusswirksamen Gebirgsbereich für radioaktive Abfälle mit einem gering durchlässigen Wirtsgestein $\left(k_{f}<10^{-10} \mathrm{~m} \mathrm{~s}^{-1}\right.$, StandAG §23, 5) und eine langfristige Abdichtung durch Barrierematerialien (EndlSiAnfV, 2020; ESK, 2019). Die potenziellen Wirtsgesteine Ton und Steinsalz sowie die betrachteten Barrierematerialien Bentonit und verdichteter Salzgrus weisen Durchlässigkeiten im Bereich von $k_{f} \sim 10^{-16} \mathrm{~m} \mathrm{~s}^{-1}\left(K \sim 10^{-21} \mathrm{~m}^{2}\right)$ auf. Diese 
niedrigen Werte legen nahe, dass die advektive Strömung genauso langsam ist wie der diffusive Stofftransport. Die Ermittlung einer solch geringen Permeabilität mit ausreichender Genauigkeit stellt eine Herausforderung für die Messanordnungen und entsprechenden Fehlerbewertungen dar.

Methoden. Niedrigpermeabilitätsbestimmungen erfolgen häufig mittels transienter Verfahren, z.B. durch Überwachung kontrollierter Änderungen des Fluiddrucks bei: (1) Druckabfall und (2) Oszillationspulstests. Beim ersten Verfahren wird die Permeabilität von der Zeit abgeleitet, die von der Probe zum Ausgleich von Druckunterschieden benötigt wird. Beim 2. Verfahren werden die Phasenverschiebung und die Amplitudendämpfung kontrollierter, durch die Probe laufender Druckimpulse beobachtet. Jede Permeabilitätsmessung muss nachbearbeitet werden, z. B. bezüglich (1) materialintrinsischer Kontrollen (Sättigungszustand, Lagerfähigkeit, Kompressibilität der Flüssigkeiten usw.), (2) Umgebungskontrollen (Temperatur, Begrenzungsdrücke usw.) und (3) theoretischer Überlegungen (Klinkenberg-Korrektur, Multiphasenbenetzungswinkel usw.).

Salze. Bis zu einem $K$-Wert von $10^{-19} \mathrm{~m}^{2}$ wurde eine Porosität-Durchlässigkeits-Beziehung gefunden (z. B. Popp et al., 2007). Testflüssigkeiten waren NaCl-Sole, Öle, He und $\mathrm{N}_{2}$ als Flüssigkeit. Laut aktueller Forschung könnte ein kritisch niedriger Permeabilitätswert mit der sog. „Perkolationsschwelle“ assoziiert werden, die die Mindestanforderungen an ein vernetztes Porensystem definiert (z. B. DAEF, 2016).

Tone. Eine große Herausforderung stellen die lange Dauer der Probensättigung (mitunter mehrere Monate) und des Druckausgleichs (oft Tage) sowie die präzise, temperaturkompensierte Messung und die Bestimmung der Lagerfähigkeit der Proben (z. B. Winhausen et al., 2021) dar. Prüfflüssigkeiten sind üblicherweise Mischungen, die das Porenwasser des Gesteins nachahmen.

Geotechnische Barrierematerialien. Der durchgeführte Permeabilitätstest ähnelt dem oben bei Salz und Ton erwähnten Verfahren. Jedoch weisen beide Barrierematerialien, Salzgrus und Bentonit, früh nach der Einlagerung eine signifikante Permeabilität auf. Dies ist von Vorteil, da dadurch unerwünschte Korrosionsgase aus der Behälterverwitterung austreten können. Im Lauf der Zeit nimmt die Durchlässigkeit um Größenordnungen $\mathrm{ab}$, und die Barrieren werden langfristig dicht. Diesbezüglich erwies sich die Bestimmung des Gaseintritts-/ Durchtrittsdrucks als nützlich (z. B. Rothfuchs et al., 2007).

Abbildung 1 zeigt eine vorläufige Sensitivitätsanalyse als Beispiel für Druckabnahmemessungen. Sie legt nahe, dass der Druckausgleichsterm (c) und damit die Prüfdauer am empfindlichsten für die Berechnung der geringen Permeabilität sind. Aufgrund der großen Variation der (repräsentativen) Material- und Umweltkontrollen sind Permeabilitätsmessungen jedoch komplex. Dieser Workshop zielt darauf ab, Diskussionen über die Unsicherheit und die Sensitivität der beeinflussenden Kontrollen anzuregen, um daraus einen Leitfaden für bewährte Permeabilitätsmessungen erstellen zu können.
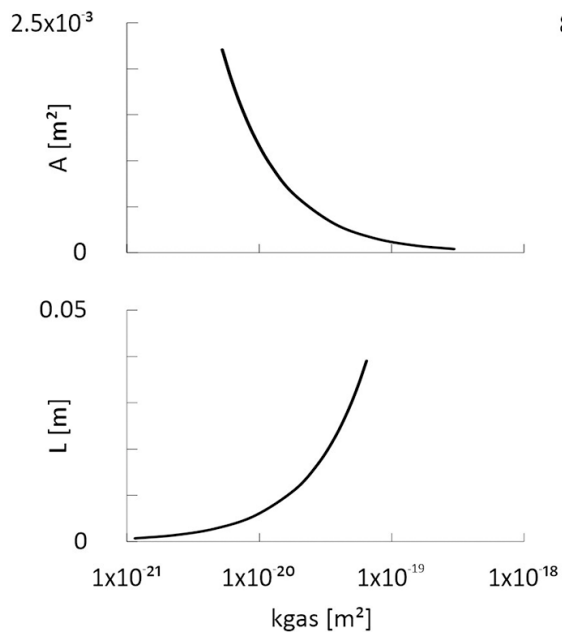
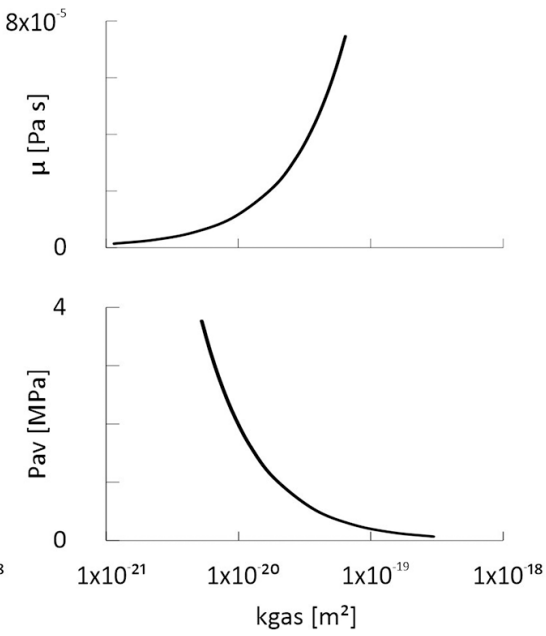
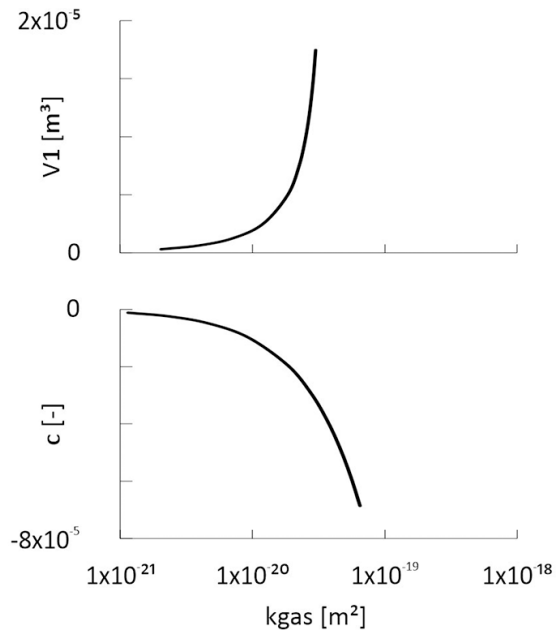

Figure 1. The sensitivity of selected input parameters for permeability estimation using the pressure decay method. $\mu=$ Dynamic viscosity of the test fluid, $\mathrm{V} 1=$ pressurized upstream volume, $\mathrm{Pav}=$ average pore pressure, $A=$ cross-sectional area of specimens, $L=$ sample length, $c=$ parameter of pressure equilibration. 


\section{References}

DAEF, Deutsche Arbeitsgemeinschaft Endlagerforschung: Kurzstellungnahme zur Veröffentlichung "Deformationassisted fluid percolation in rock salt", available at: http: //www.daef2014.org/assets/daef-science_2016-04_web-1-.pdf (last access: 28 June 2021), 2016.

ESK, Entsorgungskommission: Stellungnahme "Sicherheitskonzeptionelle Anforderungen an das Barrierensystem eines Endlagers für hochradioaktive Abfälle und deren Umsetzbarkeit”, ESK-Geschäftsstelle beim Bundesamt für kerntechnische Entsorgungssicherheit, Berlin, 2019.
Popp, T., Wiedemann, M., Böhnel, H., and Minkley, W.: Untersuchungen zur Barriereintegrität im Hinblick auf das EinEndlager-Konzept, Abschlussbericht zum Forschungsvorhaben SR 2470, Institut für Gebirgsmechanik GmbH, Leipzig, 2007.

Rothfuchs, T., Jockwer, N., and Zhang, C.-L.: Self-sealing barriers of clay/mineral mixtures - The SB project at the Mont Terri Rock Laboratory, Phys. Chem. Earth Pt. A/B/C, 32, 108-115, https://doi.org/10.1016/j.pce.2006.03.017, 2007.

Winhausen, L., Amann-Hildenbrand, A., Fink, R., Jalali, M., Khaledi, K., Hamdi, P., Urai, J. L., Schmatz, J., and Amann, F.: A comparative study on methods for determining the hydraulic properties of a clay shale, Geophys. J. Int., 224, 1523-1539. https://doi.org/10.1093/gji/ggaa532, 2021. 